\title{
From Digital to Computational Pathology and Integrated Diagnostics: The Future of Histopathology
}

\author{
Muhammed Mubarak \\ Department of Histopathology, Sindh Institute of Urology and Transplantation (SIUT), Karachi, Pakistan
}

Among the various branches of clinical pathology, histopathology has been the most conservative and slowest in adapting to automation and digitisation in this era of information technology (IT). It is still largely a manual and qualitative science; and heavily dependent on the opinion of medical experts. ${ }^{1}$ The last attribute of it imparts subjectivity to the field. One of the main reasons for its slow adaptation to automation is the fact that several, manual and largely independent steps, are involved in the preparation of material for visualisation under the microscopes by the pathologists and beyond, which, on a cursory look, seem unfit for automation and digitisation. However, there is one other example of a medical diagnostic field with similar objections and reservations in the beginning; but which, during recent past, has undergone successful digitisation, i.e. radiology. There are many similarities between radiology and histopathology: both are used for making diagnosis, both are subjective, and both involve images. Nowadays, radiology has been successfully transformed into filmless field in most parts of the world. It is now the turn of histopathology to convert into slideless arena and the change is on the horizon.

Digital pathology (DP) is defined as the practice of pathology using digital images of whole microscopic sections on computers rather than under the microscopes as is done in traditional histopathology, such that computers serve, as workstations for pathologists..$^{2-4}$ It has become possible in recent years by the development of whole-slide imaging (WSI) scanners, which capture high-resolution images of microscopic sections at a very high speed and at high magnification. They transform the glass slides into digital slides, which are immediately archived in computer systems and made accessible to all concerned. The digital slides can be efficiently stored, accessed, analysed, retrieved and shared with pathologists or scientists from across the world via internet, using slide viewing and management softwares. In DP, the glass slides are prepared in much the same way as in conventional microscopy; but instead of examining the slides under a microscope, the slides are visualised on a computer screen.

Correspondence to: Prof. Dr. Muhammed Mubarak, Department of Histopathology, Sindh Institute of Urology and Transplantation (SIUT), Karachi, Pakistan

E-mail: drmubaraksiut@gmail.com

Received: January 09, 2021; Revised: January 15, 2021;

Accepted: January 18, 2021

DOI: https://doi.org/10.29271/jcpsp.2021.01.2
The pathologist can navigate the whole section at various magnifications and measure, analyse and annotate any feature, using different types of software. One of the main advantages of DP involves acquisition of objective measurements of morphological, histochemical, and immunohistochemical features of microscopic sections, augmenting both the amount and quality of data obtained from histologic evaluations of sections by image analysis and artificial intelligence (AI) methods. The later falls in the domain of computational pathology (CP), which is defined as a branch of pathology that involves extraction of information from DP images in combination with their associated meta-data, typically using Al methods such as machine learning (ML) and deep learning (DL) to achieve objective and standardised evaluations of pathology material. Thus, CP may be defined as the "omics" or "big data" approach to pathology, wherein multiple sources of patient information including pathology image data and patient meta-data are combined to extract patterns and analyse features. ${ }^{5}$ $\mathrm{ML}$ and $\mathrm{DL}$ are types of Al-based computational methods, commonly used in CP, which are able to "learn" how to perform tasks based on examples or patterns. ${ }^{6} \mathrm{~A}$ related concept is MLpowered image analysis, which allows extremely accurate image classification or segmentation of a digital image. The outputs of these computer-based tools may later be integrated into a full CP program, once these image features are correlated to other types of patient information besides the image itself. While this type of computational analysis holds great promise for a paradigm-shift in diagnosis and delivery of healthcare, multiple hurdles remain, impeding its widespread clinical use. Nevertheless, the fields of DP and CP are rapidly evolving, and will increasingly transform the practice of histopathology, permitting it to mature toward a quantitative science. Continuing advancements in high throughput laboratory and computational technologies are serving as a driving force for the creation of conducive environment for CP. A new discipline of "integrated diagnostics" is also rapidly emerging as combining radiology, pathology and laboratory tests with patient meta-data and advanced IT to make more quick, efficient, objective, and accurate diagnosis; and to save economic and human resources. DPand CP will be integral parts of thisintegrated diagnostic system. ${ }^{5-8}$ The ultimate goal of all these innovations in pathology is to achieve optimal patient-care, especially in cancer patients.

The digital slides can be evaluated and interpreted independent of microscopes in the hospital laboratory, at home or at remote 
places via internet, giving autonomy and flexibility to pathologists, a need of the hour, given the prevailing pandemic of novel coronavirus disease 2019 (COVID-19), which has posed serious threats to the way we live and work. Although, the use of DP and $\mathrm{CP}$ is still in its early stage, it has the potential to revolutionise the field of histopathology. DP is already changing the workflows of many laboratories in developed countries of the world. $^{3-6}$

Well established applications of WSI include rapid transmission of digital images for telepathology and collaborations, standardisation and distribution of pathologic materials for education, research, publishing, slide archiving, and image analysis. However, its widespread use for making primary diagnosis in clinical laboratory is still in primitive stage. Recently, a breakthrough has been achieved for these innovative techniques as FDA has approved the use of WSI for making primary diagnosis in surgical pathology in USA. ${ }^{8}$

There are several challenges in implementing DP and CP on a larger scale, particularly in developing countries, due to technical, logistical, and financial reasons. It is a gigantic and costly task for emerging economies. There are hurdles in its smooth integration with existing IT infrastructure and workflows in the laboratories in resource-constrained settings. ${ }^{4}$ However, given the enormous long-term benefits, it is worth implementing, as in the long-term, the cost-benefit ratio is favourable for DP and CP. Before this dream is realised, there is a need to generate digital-quality pathology material by using standard equipment and reagents. It is important for obtaining digital quality images.

The innovation and sheer volume of whole slide images haveled to a number of image and data management challenges. One of these challenges is Laboratory Information Systems (LIS), which drive workflow and data management in most of the pathology departments, which are not well equipped to manage image level information. ${ }^{6-10}$

In conclusion, WSI, DP and CP are on the verge of revolutionising the field of histopathology; and the use of these technologies could transform pathology from a largely manual, subjective and qualitative field into one that is data-driven, objective and quantitative; and hence, more accurate, reliable and consistent in informing patient-care.

\section{REFERENCES}

1. Kazi JI, Mubarak M. Histopathology in present era. J Pak Med Assoc 2009; 59(1):1-2.

2. Jahn SW, Plass M, Moinfar F. Digital pathology: Advantages, limitations and emerging perspectives. J Clin Med 2020; 9(11):3697. doi: 10.3390/jcm9113697.

3. Higgins C. Applications and challenges of digital pathology and whole slide imaging. Biotech Histochem 2015; 90(5): 341-7. doi: 10.3109/10520295.2015.1044566.

4. Volynskaya Z, Evans AJ, Asa SL. Clinical applications of whole-slide imaging in anatomic pathology. Adv Anat Pathol 2017; 24(4):215-21. doi: 10.1097/PAP.000000000 0000153.

5. Abels E, Pantanowitz L, Aeffner F, Zarella MD, Van der Laak J, Bui MM, et al. Computational pathology definitions, best practices, and recommendations for regulatory guidance: $A$ white paper from the digital pathology association. J Pathol 2019; 249(3):286-94. doi: 10.1002/path.5331.

6. Madabhushi A, Lee G. Image analysis and machine learning in digital paathology: Challenges and opportunities. Med Image Anal 2016; 33:170-5. doi: 10.1016/j.media.2016. 06.037.

7. Aeffner F, Zarella MD, Buchbinder N, Bui MM, Goodman MR, Hartman DJ, et al. Introduction to digital image analysis in whole-slide imaging: A white paper from the digital pathology association. J Pathol Inform 2019; 10:9. doi: 10.4103/jpi.jpi_82_18.

8. Evans AJ, Bauer TW, Bui MM, Cornish TC, Duncan H, Glassy $E F$, et al. US food and drug administration approval of whole slide imaging for primary diagnosis: A key milestone is reached and new questions are raised. Arch Pathol Lab Med 2018; 142(11):1383-7. doi: 10.5858/arpa.2017-0496CP.

9. Snead DR, Tsang YW, Meskiri A, Kimani PK, Crossman R, Rajpoot NM, et al. Validation of digital pathology imaging for primary histopathological diagnosis. Histopathol 2016; 68(7):1063-72. doi: 10.1111/his.12879.

10. Pantanowitz L, Sinard JH, Henricks WH, Fatheree LA, Carter $A B$, Contis $L$, et al. College of American pathologists pathology and laboratory quality center. Validating whole slide imaging for diagnostic purposes in pathology: Guideline from the college of american pathologists pathology and laboratory quality centre. Arch Pathol Lab Med 2013; 137(12):1710-22. doi: 10.5858/arpa.2013-0093CP. 